\title{
Design and Implementation of DXF File Preview System in Computer Numerical Control Programming Technology
}

\author{
Wu Aizhi \\ College of Vehicles and Engergy \\ Yanshan University \\ Qinhuangdao, P.R.China \\ e-mail:ysuwaz@163.com \\ Zhao Haihang \\ School of Software Engineering \\ Yanshan Univeristy \\ Qinhuangdao, P.R.China \\ e-mail: ysusst@163.com
}

\author{
Li Zhiming \\ School of Software Engineering \\ Yanshan Univeristy \\ Qinhuangdao, P.R.China \\ e-mail: lizm@ysu.edu.cn
}

\begin{abstract}
With the improvement of the advanced manufacturing system integration requirement, obtaining graphic data from DXF file provided by AutoCAD software becomes more necessary. DXF (diagram interchange file) is an open vector data format, which is easy to read and analysis, and it has always been the general CAD/CAM researchers' study object. However, as the strengthen of AutoCAD function, DXF file has taken place great change on its form and content. Early versions of DXF file conversion tools can no longer be suitable for the new version of DXF file conversion. Therefore, the research and development of universal interface module is needed. The main research object of this paper is DXF and the main content is the design of the preparatory phase of the NC (Numerical Control) machine tool. This research aims to use UML (Uniform Markup Language) modeling technology to describe and analyze DXF general components, and implement it through OpenGL (Open Graphics Library) code. Eventually the primitive information stored in DXF file can be presented in the form of graphical display, and the preview function of DXF data could be realized.
\end{abstract}

Keywords-Manufacturing Industry; Computer Numerical Control; AutoCAD; DXF File; Visualization

\section{INTRODUCTION}

DXF file is a kind of graphics interchange format file, which is defined by Autodesk Company for AutoCAD and external CAD/CAM system interface [1]. With the wide application of AutoCAD in all walks of life in the world, at present, nearly all of the CAD/CAM system must possess the function of AutoCAD interface [2]. With the improvement of advanced manufacturing requirements for system integration, obtaining graphic data from DXF file is becoming increasingly necessary [3]. The text format of DXF file is easy to read and analyze, which has always been the general CAD/CAM researchers' study object. There are only $7 \%$ of CAD/CAM researchers engaged in DXF file interface development technology [4]. Therefore, the development and changes of DXF should be paid great attention by people engaged in the research and development of CAD and CAM.

Along with the strength of AutoCAD function, great changes have taken place in DXF file on its form and content. Early versions of DXF file conversion tools can no longer be suitable for the new version of DXF file conversion. Therefore, the research and development of new interface module is needed. Cutting blanking is the first step of all machining parts processing, and the essence of which is the cutting and processing of two-dimensional structure [5].

A lot of experience in production practice shows that AutoCAD is used by all production departments in preliminary design, especially in the phase of cutting material preparation and in the field of sheet metal production [6]. Therefore, the study of two-dimensional structure of DXF data acquisition and processing method has practical significance. The design and development of universal DFX data reading and visual display software system also have important application value.

Through the analysis of two-dimensional part geometry feature, this paper studies the method of obtaining twodimensional parts graphic data from the DXF file, puts forward the idea of using closed loop and tree linear table to store complex two-dimensional part geometric features [7] which can separate two-dimensional institutional characteristics from complex DXF file, and provides the processing and implementation method before and after the numerical control and implementation method of twodimensional structure of cutting based on the process features of two-dimensional plate cutting processing.

This paper introduces the development process of system with details in the view of demand analysis, general design, detailed design and system test, and briefly introduces the main characteristics of the system and the main system development tools. Finally, a prototype system was developed with visual interface. 


\section{FUCTION ANALYSIS AND OVERALL DESIGN}

DXF format is a representation method of the tag data of all the information in a contained in specific version of AutoCAD graphics file [8]. Tag data is the integer called set code in front of each data element. Values of set code show the type of subsequent data elements, and point out the meaning of data elements (or record) for a given object (or record) type. Actually, in graphics file all the information specified by users can be express in DXF file format. DXF formats used in AutoLISP and ARX application are basically the same as the format mentioned above, subtle differences only exist in some of the data set [9].

A DXF file consists of several sets and each set has two lines. The first line is set code and the second line is set value. Set code equals to the code of data type which is ruled by CAD graphic system, while the set of value is specific value, the two of which combined together representing the meaning and value of a data [10]. Set code is a nonnegative integer no more than three digits, and the set value is determined by the type of set code. Each set code has specific meaning. The meaning of some code is fixed, and some set code has multiple meanings due to different applications. Therefore, they should be analyzed specifically. The general structure of DXF file is shown in Fig. 1.

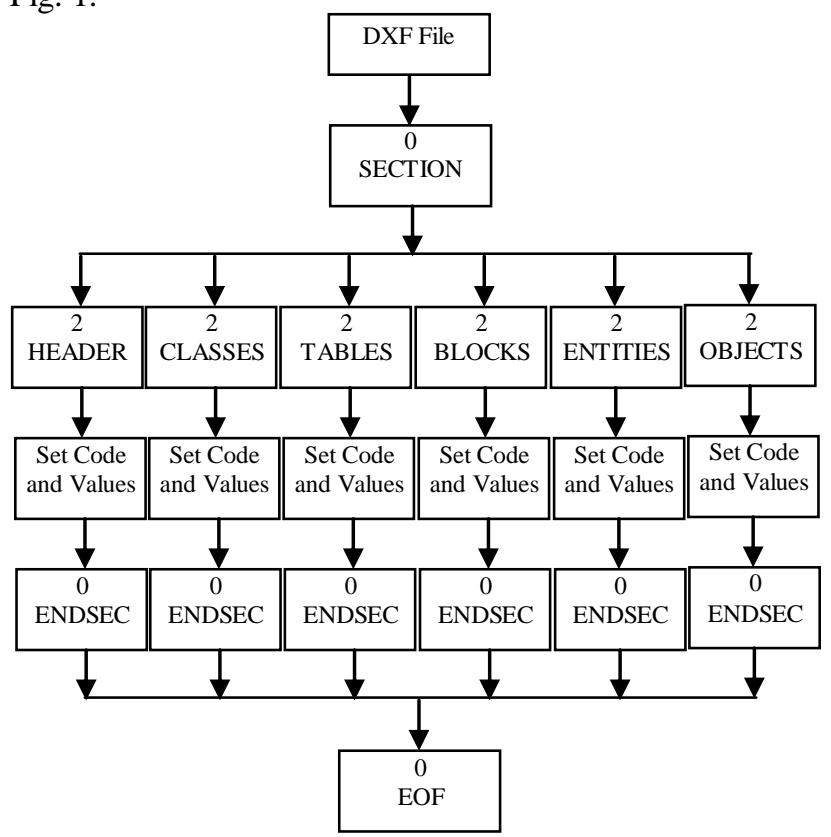

Figure 1. General structure of DXF file

Due to the complexity of DXF file structure, in practice, in order to extract entity information of graphic, many items in DXF file could be omitted, and the description of corresponding geometric shapes could be completed as long as accessing the layer table, block and entity [11].

According to the format and reading requirements of DXF file, the design and development of this system is divided into 5 phases:

1) System requirement analysis and system function design, including feasibility analysis, detailed system investigation, user requirement analysis, collecting all kinds of users' requirements on system function, organizing related information data, and settling down the scope of system development.

2) Thorough understanding of DXF file format, comprehend DXF file format, make sure the primitive types and species in the file, and Establish the data structure of program.

3) Partitioning and implementation of program module. Carry out system module partition according to system function and write code accordingly so as to realize system function.

4) Security policy design. Design security model according to system function.

5) Test. Carry on system test after the development of main functions to find out design defects and errors, and then make improvement.

The whole system is divided into three system module, namely data extraction, data analysis and drawing primitive, mainly including the reading and drawing of Line, Arc, Circle, Polyline and Spline primitives, etc [12]. System overall structure is shown in Fig. 2.

Primitive information in DXF file is read by this system base on object-oriented idea, and the primitive stored in file is drawn at the same time. Detailed design and development ideas are as below:

(1) Based the idea of object-oriented program design, design the overall architecture of program through UML modeling technology [13], so as to keep better scalability and scalability of the system.

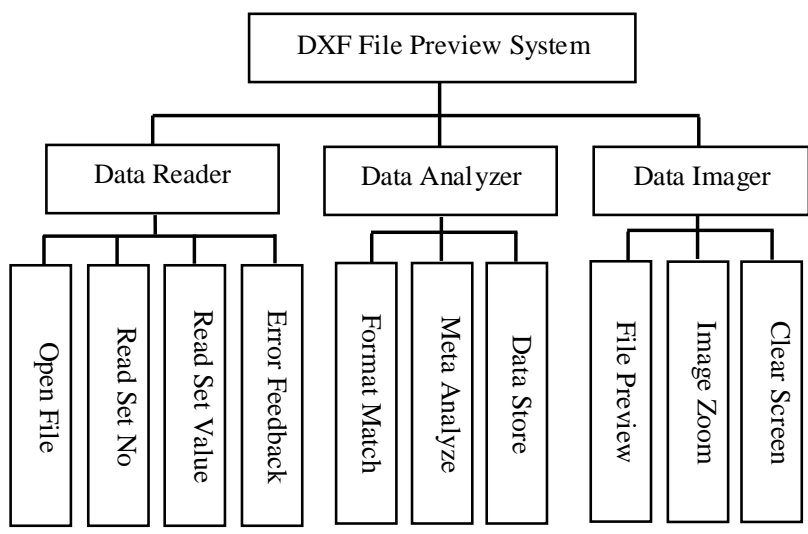

Figure 2. System overall structure

(2) Under the programming environment of Microsoft Visual Studio 2010, c \# language is used in code written and all functions are completed in this system.

(3) Through a detailed analysis of data such as set code and set data in DXF file, primitive information described in file could be read.

(4) Primitive information store in file is drawn and displayed completely through adopting OpenGL technology.

(5) Keep system scalability for after effective improvement and modification of the system.

\section{DESIGN AND ANALYSIS OF EACH MODULE}

\section{A. Data reading module}

In data extraction module, system first need to open specified DXF file, and then analyze the primitive information in file one by one according to the DXF file 
format agreement, and the specific process is as shown in Fig. 3.

\section{B. Data analysis module}

In data analysis module, system should determine which section the data belongs to based on the analysis of set code and set value in documents, to prepare for after primitives drawing. The general structure, and the specific composition of ENTITIES and Header set code and set value is shown in Fig. 4 and 5.

\section{Drawing primitive module}

In drawing primitive module, all the primitive information contained in file is drawn and displayed in the canvas by system through the OpenGL technology according to the analysis of the data analysis module, the specific process is shown in Fig. 6.

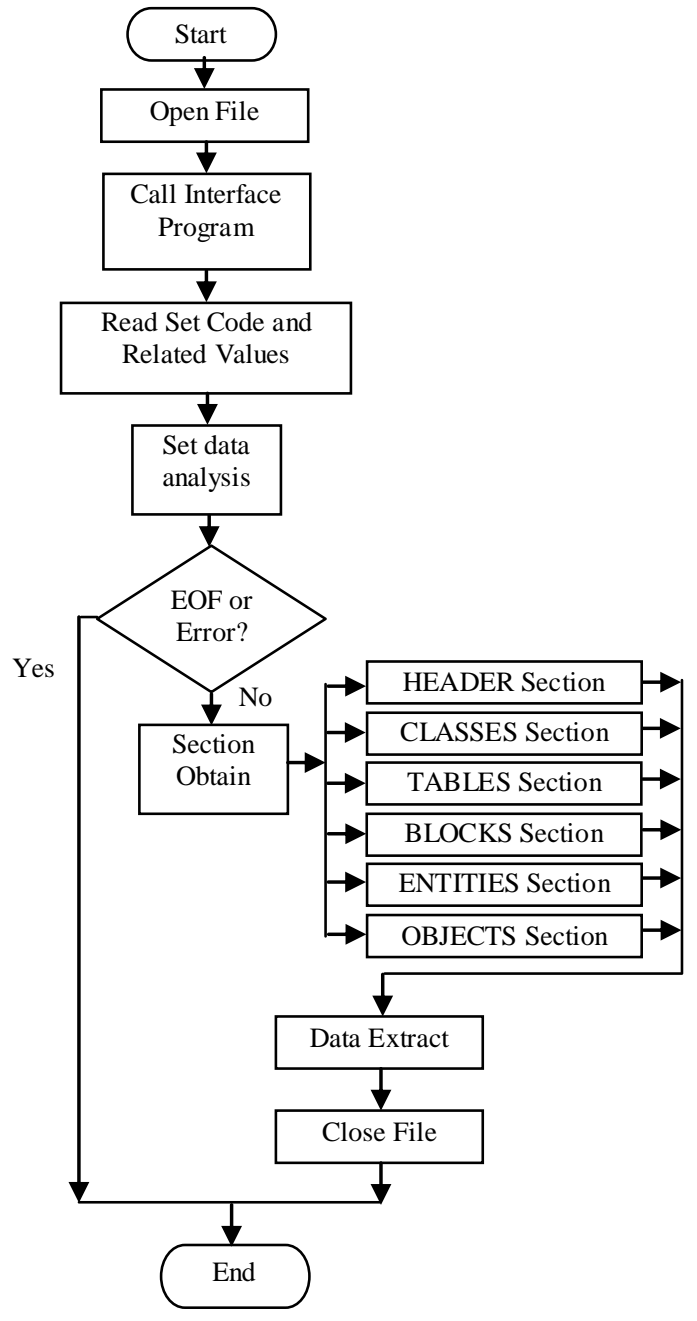

Figure 3. Data extraction flow of DXF file

This part mainly introduces the overall design of DXF file primitive preview system. It begins from the main system development work, and then makes a module division according to system function, through independent coding of each module, finally combines and realizes all the functions of system, so as to realize the flexibility and portability of the system.

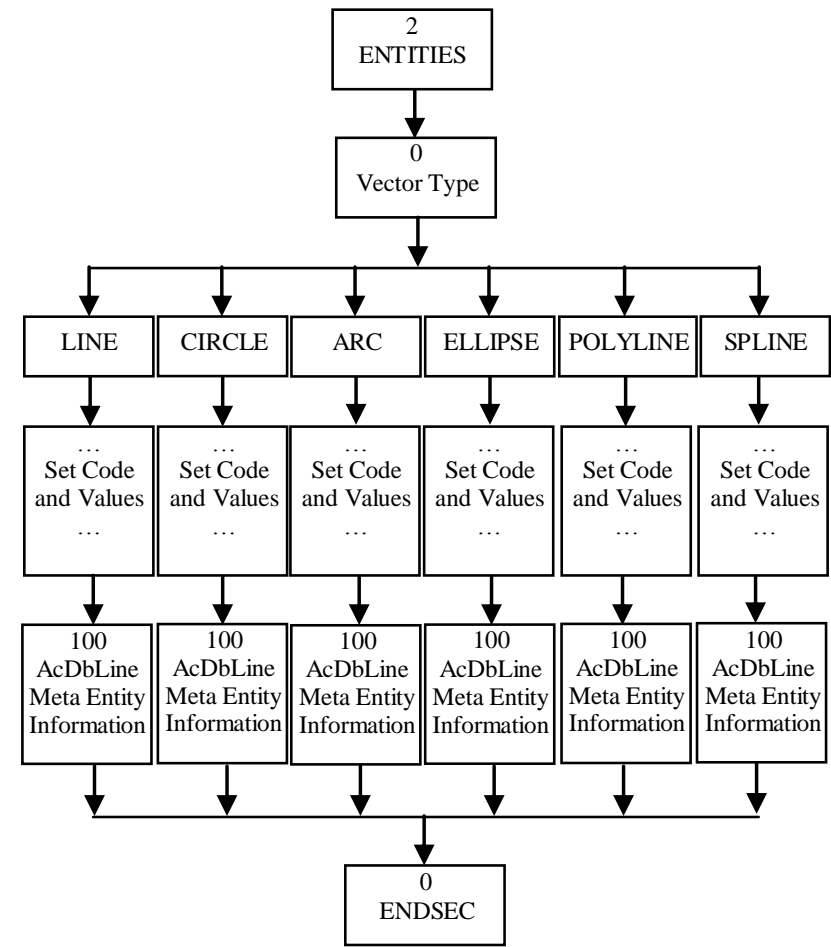

Figure 4. Set code and value sturcture of ENTITIES section

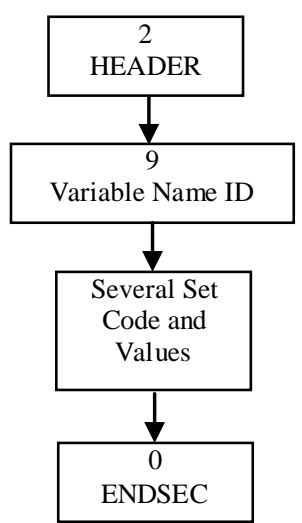

Figure 5. Set code and value composition sturcture of Header section

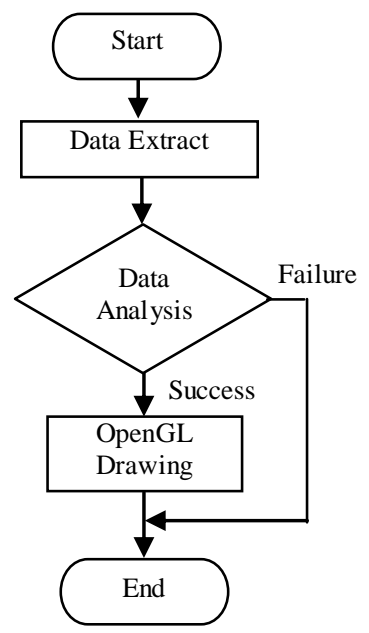

Figure 6. Primitive drawing flow 


\section{REALIZATION AND RUNNING EFFECT OF THE SYSTEM}

The design of this system code follows the principles as below [14]:

(1) Follow the development process and write code under the guidance of design.

(2) Code written aims to realize the function and performance of design, requires correct completion of design function required, and meet the design performance.

(3) Good structure is required so as to guarantee good encapsulation and reduce the coupling of application program.

(4) Readable, easy to understand; convenient to test.

(5) Easy to use and maintain, good modification and expansion, good portability/ reusability.

(6) Occupy less resources and complete task with a low cost.

(7) Try to improve the efficiency of code execution without reducing the readability of program.

Primitive in DXF file is drawn (preview) by OpenGL, some functions of OpenGL need to be called in this module to implement some function. The original file of DXF and primitive drawing module interface diagram is shown in Fig. 7 and 8.

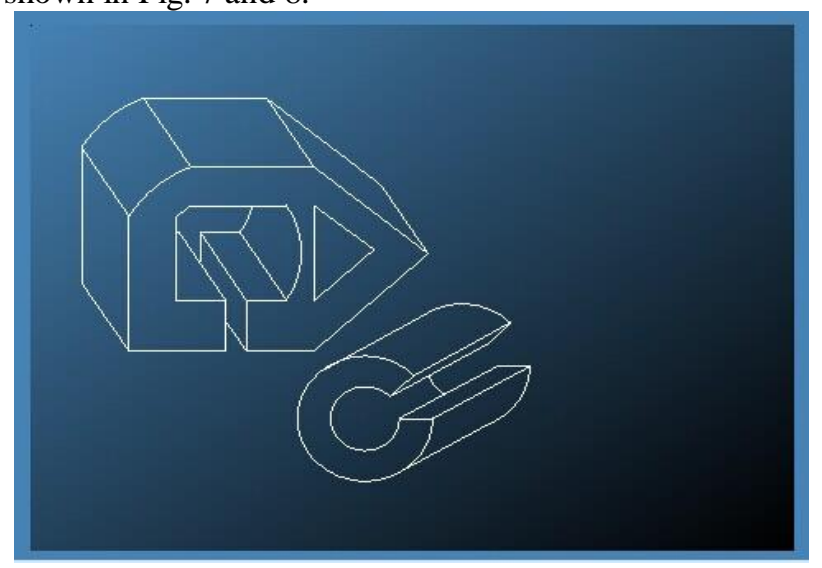

Figure 7. Original file of DXF selected

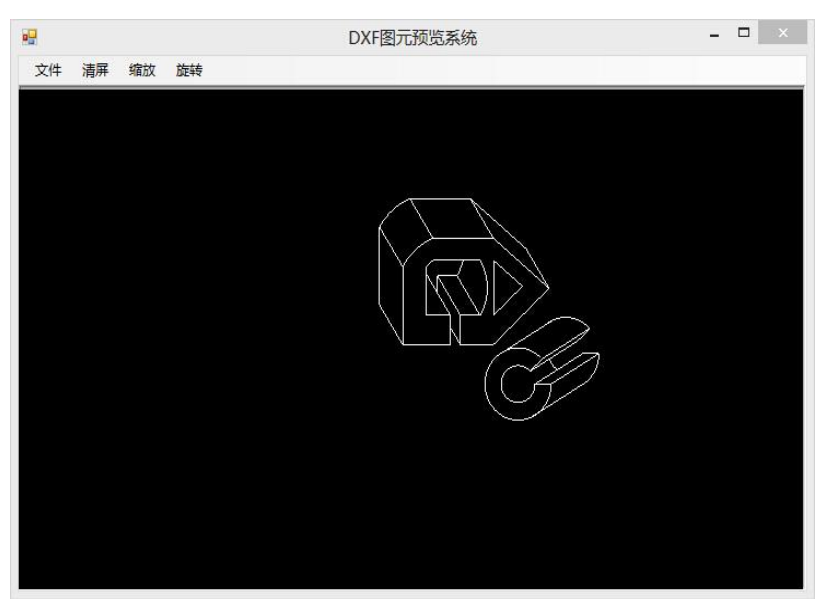

Figure 8. Primitive drawing module interface diagram

In this chapter, a simple and convenient user interface is designed, and the main functions are realized. basic coding is completed at the same time, and some basic functions of DXF file primitive preview system are realized.

\section{CONCLUSIONS}

From research background, topic selection, feasibility study, demand analysis to the overall and detailed design, finish of the whole process of the whole system, this paper introduces DXF file primitive preview system in detail.

The design is developed in Microsoft Visual 2010 environment, OpenGL technology is used, graphical interface is adopted, which provide convenient and efficient user experience. This system adopts single interface style, which is clear and direct to operate, and the man-machine interaction is suitable for the user.

The main design task expected is completed basically and the whole system can run smoothly after testing and constant improvement. Deficiencies still exist in this system, and subsequent developments are needed in following research and study.

\section{ACKNOWLEDGMENT}

This work is supported by the Project of Hebei Science and Technology Research and Development Plan (No. 13211828, The Research of Open Precision Laser Cutting Computer Numerical Control System Software Architecture), and National Natural Science Foundation of China (No. 61303130).

\section{REFERENCES}

[1] Zhang Hongxin, Wang pengfei, Youbo. AutoCAD Information Read and Data Conversion on Nc Machining Platform [J]. Journal of Harbin Institute of Technology,2002, 7(3):74-77.

[2] Cheng Yongping. Introduction of AutoLISP's Secondary Development of AutoCAD [J]. Shanxi Architecture, 2004, 30(16): 11-12.

[3] Liu Meng. Optimization Storeage Technology and Application Research of Parts Graphics Based on DXF File [M]. Mechnical Industry Press, Beijing, 2009.

[4] Yuan Yuling. The Application Environment and Development Tools of CAD Technology [J]. Shanxi Architecture, 2004,30(23): 47-48.

[5] Yu Shilin, Zhu Guobao. Research of General Nc Automatic Programming System [J]. Journal of Wuhan Institute of Technology,2003,(5): 725-727.

[6] Tong Bing. Mechanical Technology Base[M]. Tsinghua University Press, Beijing, 2012.

[7] Wang Xingbo, Li Shengyi. Realization of 3D Recovery in DXF File [J]. Computation Technology and Automation, 2006, 15(1): 15.

[8] Zhang MingJun. Research on Nc Linear Cutting Automatic Programming System [J]. Modern Manufacturing Engineering, 2000, 11: 14-15.

[9] Noack R. Converting CAD Drawings to Product Models, Licentiate Thesis [D]. Department of Real Estate and Construction Management, Royal Institute of Technology, Stockholm, Sweden. 2001.

[10] Wang Xingbo, Wan Bin. The Acquisition and Numerical Control Processing of two dimensional parts DXF Data.[J]. Manufacturing Automation, 2003, 6: 34-36.

[11] T.R. Jackson. Analysis of Functionally Craded Materiall Object Representation Methods [D]. PhD thesis, M.I.T., Cambridge, 2001.

[12] Chen Yuanyan, Zhang Xiaojing. Practical Technology of Computer Graphics [M]. Science and Technology Press, Beijing, 2011.

[13] H.Liu. Algorithms for Design and interrogation of Functionally Graded Material Solids [D]. Master's thesis,Massachusetts institute of Technology, Cambridge, 2011.

[14] Wang Yang. Automatic Programming System of NC Machine Tool Graphics [J]. Machine Tool and Hydraulic Pressure, 2010, (3): 1215. 